\title{
Mass of quasi-particles
}

\author{
Dzedolik I.V.
}

Taurida V. Vernadsky National University, 4 Vernadsky Ave., 95007

Simferopol, Ukraine, e-mail: dzedolik@crimea.edu

Received: 07.06.2007

\begin{abstract}
Spontaneous symmetry breaking in the system "dielectric mediumelectromagnetic field" is considered. The appearance of massive quasi-particles in a boundless dielectric medium, a dielectric resonator and a dielectric waveguide is shown. The mass of the quasi-particles arises because they move in the medium or the waveguide with the velocity less than the speed of light in vacuum, as a result of interaction of electromagnetic field and the medium.
\end{abstract}

Keywords: dielectric medium, dielectric resonator, dielectric waveguide, spontaneous symmetry breaking, cnoidal wave, quasi-particle, polariton, mass.

PACS: 42.25.Bs, 42.50.Ct, 42.65.-k

\section{Introduction}

Propagation of electromagnetic beam in dielectric media may be considered as a reradiation of the falling electromagnetic wave by the polarization wave, which is in general nonlinear. Otherwise, electromagnetic wave propagating in a medium may be considered as a flow of quasi-particles called as polaritons. They represent bound states of the electromagnetic field and the polarization wave [1, 2]. In both the classical and quantum "dielectric medium-electromagnetic field" models, their symmetry can spontaneously break, thus giving rise to Goldstone massless bosons and massive quasiparticles [3]. Spontaneous symmetry breaking occurs in the system if the symmetry of its ground (vacuum) state in the presence of field is lower than the initial symmetry in the absence of the field. As mentioned above, this produces the quasi-particles with the mass $m=\hbar c^{-2} \omega(k=0) \neq 0$ and the massless collective excitations (bosons), with their frequencies decreasing down to zero $(\omega(\mathbf{k}) \rightarrow 0)$ as the wave-vector tends to vanish $(\mathbf{k} \rightarrow 0)$. The collective excitations compensate the initially broken symmetry of the system. Therefore, the number of spectrum branches of the Goldstone bosons corresponds to the number of missed independent elements of the continuous Lagrangian symmetry group [3].

The quasi-particles are excitations over the vacuum state of linear and nonlinear

Ukr. J. Phys. Opt. 2007, V8, №4 
systems. Solitary or periodic cnoidal waves assigned to the quasi-particles can arise in boundless dielectric media, dielectric resonators and dielectric waveguides such as optical fibres [4-10]. Under certain conditions, the quasi-particles can take a shape of 3D solitary waves $[3-6,9,10]$. The field in the form of solitary waves, with the particle-like properties, can exist in systems, which are capable of giving rise to topological states, with global minima of potential energy occurred where the field is not equal to zero. The conditions for generating particle-like excitations are specified by relationships between the field parameters and the dispersion characteristics of the medium. We consider how quasi-particles in the boundless and bounded (both linear and nonlinear) dielectric media acquire a mass.

The origin of mass of a particle is still a pending physical problem [11]. The electromagnetic field in vacuum propagating with the velocity $\mathrm{c}$ of light is the massless field, i.e. the mass of photon is equal to zero in vacuum [3]. This follows immediately from the Maxwell dispersion law $\left(\omega^{2} / \boldsymbol{c}^{2}-\mathbf{k}^{2}=0\right)$, where $\omega$ is the frequency of the wave and $\mathrm{k}$ the wave vector. From this equation we find the impulse of the field $(\mathbf{p}=\hbar \mathbf{k})$, which is connected with its energy $\widetilde{\boldsymbol{E}}=\hbar \omega$ by the expression $\widetilde{E}=p c$.

The velocity of massive particle is always less than the velocity of light in vacuum $(v<c)$ and the relativistic relationship between the energy and impulse ( $\widetilde{E}=\sqrt{c^{2} \mathbf{p}^{2}+m^{2} c^{4}}$ ) includes the mass of the particle $\mathrm{m}$. The mass of a body always depends on the interaction of its particles [11]. The mass of a quasi-particle depends on the interaction of electromagnetic field and medium, too. The mass characterizes inertial and gravitational properties of the quasi-particle, i.e. the medium becomes heavier as the electromagnetic wave propagates through it $[7,12]$.

De Broglie has been the first who paid attention to occurrence of massive quasiparticles in a bounded space like a waveguide [13]. Feynman [14] has compared the relativistic relationship between the energy and impulse of a massive particle $\widetilde{E}^{2}=\mathbf{p}^{2} c^{2}+m^{2} c^{4}$ to the dispersion law $\omega^{2}=c^{2} \mathbf{k}^{2}+c^{2} \pi^{2} / a^{2}$ for the modes of rectangular waveguide with the length a, where the mass of the quasi-particle should be equal to $m=\hbar \pi / c a$. In a dielectric waveguide, the frequency of electromagnetic field depends on the propagation constants of modes $\beta_{\ell n}$, which is evident from the transcendental dispersion equation [15]. The mass of the mode is obviously not allocated, but it is possible to find the longitudinal impulse of the quasi-particle $\hbar \beta_{\ell n}$ in the dielectric waveguide from this dispersion equation.

With the appearance of quantum mechanics, it has become possible to treat the quantum particles as waves. On the other hand, there have been attempts to describe classical waves as fluxes of quasi-particles. The relativistic connection of the impulse and energy yields the mass spectrum of quasi-particles propagating in dielectric medium with the velocity less than the speed of light in vacuum. 


\section{Mass of photon in resonator}

The mass of electromagnetic field $m=\widetilde{E} / c^{2}$ existing in unclosed resonator like FabryPerot one appears due to the standing waves, because the energy transition does not take place in this case $[7,12]$. The components of the electric $\left(e_{x}\right)$ and magnetic $\left(h_{y}\right)$ fields in the Fabry-Perot resonator are superpositions of the direct and opposite plane waves with the amplitude $\mathrm{e}_{0}$ in vacuum $(\omega=c k)$ :

$$
\begin{aligned}
& e_{x}=e_{0} \sin (\omega t-k z)+e_{0} \sin (\omega t+k z)=2 e_{0} \sin (\omega t) \cos (k z), \\
& h_{y}=c \int d t \frac{\partial e_{x}}{\partial z}=2 e_{0} \cos (\omega t) \sin (k z) .
\end{aligned}
$$

We find the energy density $\mathrm{w}$ of the standing wave in the resonator as

$$
w=\frac{1}{8 \pi}\left(e_{x}^{2}+h_{y}^{2}\right)=\frac{e_{0}^{2}}{2 \pi}\left[\sin ^{2}(\omega t) \cos ^{2}(k z)+\cos ^{2}(\omega t) \sin ^{2}(k z)\right] .
$$

The average of the field period energy, which is confined between the nearest minima, is equal to

$$
\overline{\tilde{E}}=\frac{e_{0}^{2} \lambda}{8 \pi} S,
$$

where $\mathrm{S}$ is the square of mirrors. The quantity $\overline{\tilde{E}}$ characterizes the average energy of electromagnetic field located along the resonator length on the half-wave length $\Delta z=\lambda / 2$ and does not move in space. On the other hand, the energy of electromagnetic field mode in the resonator expressed in terms of the number of photons $\mathrm{N}$ is equal to

$$
\tilde{E}=\tilde{E}_{1}\left(N+\frac{1}{2}\right)
$$

where $\tilde{E}_{1}$ is the single photon energy. We can find the energy of single photon in the resonator, while equating Eqs. (3) and (4) $\left(\overline{\tilde{E}} N_{\min }=\tilde{E}\right.$, where $N_{\min }=L / \Delta z$ is the number of located energy bunches and $\mathrm{L}$ the resonator length):

$$
\tilde{E}_{1}=\frac{e_{0}^{2}}{4 \pi(N+1 / 2)} V
$$

with $\mathrm{V}=\mathrm{SL}$ being the volume of the resonator. Then the mass of quasi-particle termed a "resonator photon" should be equal to

$$
m=\frac{\tilde{E}_{1}}{c^{2}}=\frac{e_{0}^{2}}{4 \pi c^{2}(N+1 / 2)} V .
$$

The quasi-particle "resonator photon" gets its mass in the motionless frame of reference associated with the resonator, since, as a result of interference of the waves reflected from the mirrors, the standing wave does not transfers its energy. The standing 
waves in waveguides form a cross mode field distribution. The longitudinal field distribution of the waveguided mode is featured by the travelling wave. The waveguided modes could be represented as a flow of quasi-particles. The quasi-particles have the masses if their velocity in the waveguide remains less than the vacuum light speed.

The photon in the resonator with flat mirrors differs from that in the free space, represented by the plane wave, by a presence of mass and cross structure of the field. One can see that the mass of electromagnetic field decreases in the resonator with energy losses for that field, as if for a body with variable mass. The analogy is well observed for such a classical macroscopic object as a mode of electromagnetic field in metal or dielectric resonators, as well as in the waveguides, with the behaviour of a quantum microscopic object like a particle in potential well. So, the ideal metal waveguide is treated as a two-dimensional well of an unlimited depth, while the dielectric waveguide as a two-dimensional well of a limited depth [7].

\section{Wave packet in dielectric medium and in waveguide}

Let us consider the requirements for occurrence of a quasi-particle mass in a boundless dielectric medium [10]. The mass of the particle may be determined from the relativistic relationship between its energy and impulse, $\tilde{E}=\sqrt{c^{2} \mathbf{p}^{2}+m^{2} c^{4}}$. Assuming that the energy of the wave packet with the spectral width of $\Delta \omega$ is $\tilde{E}=\hbar\left(\omega_{q}+\Delta \omega\right)$ and its impulse is $\mathbf{p}=\hbar \mathbf{k}$, we obtain the mass of the wave packet as a quasi-particle: $m=\left[\hbar^{2}(\omega+\Delta \omega)^{2}-c^{2} \hbar^{2} k^{2}\right]^{1 / 2} / c^{2}$. This mass may be found experimentally by measuring independently the energy (spectrum) $\tilde{E}=\hbar(\omega+\Delta \omega)$ and the impulse (or the wavelength) $p=2 \pi \hbar / \lambda$ in the given medium.

When the electromagnetic wave packet propagates through a dispersive (and absorbing [13]) dielectric medium, the period-averaged temporal variation of its energy density may be classically represented as

$$
\frac{\partial \bar{w}}{\partial t}=\frac{1}{16 \pi}\left[i \omega\left(\varepsilon^{*}-\varepsilon\right) \mathbf{E}_{0} \mathbf{E}_{0}^{*}+\frac{d\left(\omega \varepsilon^{*}\right)}{d \omega} \mathbf{E}_{0} \frac{\partial \mathbf{E}_{0}^{*}}{\partial t}+\frac{d(\omega \varepsilon)}{d \omega} \mathrm{E}_{0}^{*} \frac{\partial \mathbf{E}_{0}}{\partial t}+\frac{\partial \mathbf{B}_{0}^{*} \mathbf{B}_{0}}{\partial t}\right],
$$

where $\mathbf{E}=\mathbf{E}_{0}(t, \mathbf{r}) e^{-i \omega t}$ and $\mathbf{B}=\mathbf{B}_{0}(t, \mathbf{r}) e^{-i \omega t}$ are the electromagnetic field vectors with their amplitudes $\mathbf{E}_{0}(t, \mathbf{r})$ and $\mathbf{B}_{0}(t, \mathbf{r})$ slowly varying in time, the symbol * means complex conjugation, and $\varepsilon=\varepsilon^{\prime}+i \varepsilon^{\prime \prime}$ is the complex permittivity of the medium. When deriving Eq. (7), Taylor expansion of the operator $f_{1}(\omega+\Delta \omega)=\frac{\partial}{\partial t} \varepsilon$ in the spectral width $\Delta \omega<<\omega$ is used and the fact that the amplitudes vary slowly in time, i.e. $\mathbf{E}_{0}(t, \mathbf{r})=\mathbf{E}_{0 \Delta \omega}(\mathbf{r}) e^{-i \Delta \omega t}$, is taken into account [16]. The average energy density of the field in the medium must increase with time in the conditions of thermodynamic equilibrium, and the medium can only absorb the field energy. Integration of Eq. (7) over 
the time of field action, $\int_{t_{0}}^{t} d t^{\prime}$, yields the average energy density in the field-medium system,

$$
\bar{w}=\frac{1}{16 \pi}\left[\omega \varepsilon^{\prime \prime} \mathbf{E}_{0}^{*} \mathbf{E}_{0} \Delta t+\frac{d\left(\omega \varepsilon^{\prime}\right)}{d \omega} \mathbf{E}_{0}^{*} \mathbf{E}_{0}+\mathbf{B}_{0}^{*} \mathbf{B}_{0}\right],
$$

where $\Delta t$ is the time of field-medium interaction.

Let us find stable states of a cubically nonlinear field-medium system. The permittivity of the medium is represented as a function of field energy density, $\varepsilon^{\prime}=\varepsilon_{1}+4 \pi \chi_{3} \mathbf{E}_{0}^{*} \mathbf{E}_{0}$, where $\varepsilon_{1}=1+4 \pi \chi_{1}$ is the linear permittivity. Then the average field energy density may be expressed in the form

$$
\bar{w}=\frac{1}{16 \pi}\left[\left(\varepsilon_{1}+\omega \frac{d \varepsilon_{1}}{d \omega}+\omega \varepsilon^{\prime \prime} \Delta t\right) \mathrm{E}_{0}^{*} \mathrm{E}_{0}+4 \pi \chi_{3} \mathbf{E}_{0}^{*} \mathbf{E}_{0} \mathbf{E}_{0}^{*} \mathbf{E}_{0}+\mathbf{B}_{0}^{*} \mathbf{B}_{0}\right] .
$$

We take the energy of the system in the linear mode of field propagation:

$$
\bar{w}_{0 L}=\frac{1}{16 \pi}\left(\tilde{\varepsilon} \mathbf{E}_{0 L}^{*} \mathbf{E}_{0 L}+\mathbf{B}_{0 L}^{*} \mathbf{B}_{0 L}\right),
$$

where $\tilde{\varepsilon}=\varepsilon_{1}+\omega \varepsilon^{\prime \prime} \Delta t+\omega d \varepsilon_{1} / d \omega$. In the normal-dispersion region, we have $d \varepsilon_{1} / d \omega>0$, the parameter $\widetilde{\varepsilon}$ is always positive $(\tilde{\varepsilon}>0)$ and the ground state of the system is symmetric. In the nonlinear mode $\left(\chi_{3} \neq 0\right)$, the ground state is also symmetric if $\widetilde{\varepsilon}>0$ and $\chi_{3}>0$ (Fig. 1). However, when $\tilde{\varepsilon}<0$ and $\chi_{3}>0$, an absolute minimum of the energy is attained $\left(\mathbf{E}_{0 m}^{*} \mathbf{E}_{0 m} \equiv \psi_{0 j}^{*} \psi_{0 j}=\tilde{\varepsilon} / 8 \pi \chi_{3}\right.$ and $\left.\mathbf{B}_{0}=\mathbf{B}_{0 L}\right)$. In this case the energy density in the ground state is $\bar{w}_{0 S B}=\frac{1}{16 \pi}\left[\mathbf{B}_{0 L}^{*} \mathbf{B}_{0 L}-\frac{\tilde{\varepsilon}^{2}}{16 \pi \chi_{3}}\right]<\bar{w}_{0 L}$ and, in particular, we

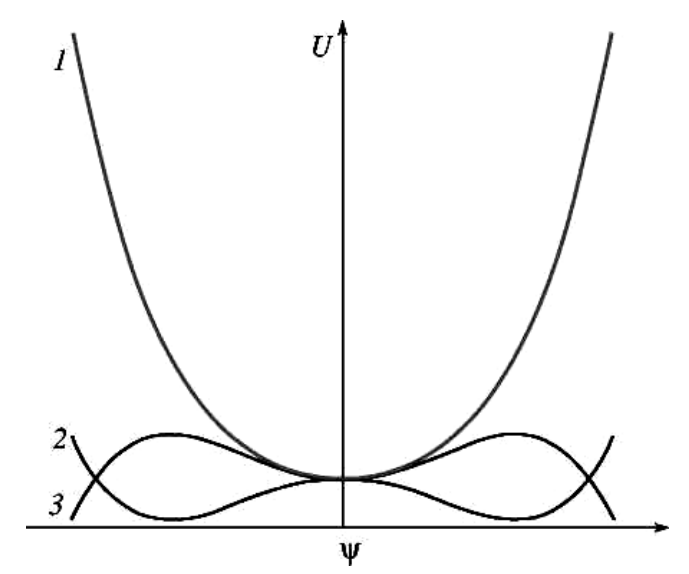

Fig. 1. Potential $U$ for a number of cases: (1) $U=a_{1} \psi^{2}+a_{2} \psi^{4}$, (2) $U=-a_{1} \psi^{2}+a_{2} \psi^{4}$ and (3) $U=a_{1} \psi^{2}-a_{2} \psi^{4}$. 
have $\bar{w}_{0 S B}=0$ at $\mathbf{B}_{0 L}^{*} \mathbf{B}_{0 L}=\tilde{\varepsilon}^{2} / 16 \pi \chi_{3}$. In the opposite case $\left(\tilde{\varepsilon}>0\right.$ and $\left.\chi_{3}<0\right)$ the energy density shows a maximum: $\bar{w}_{0 A}=\frac{1}{16 \pi}\left[\mathbf{B}_{0 L}^{*} \mathbf{B}_{0 L}+\frac{\tilde{\varepsilon}^{2}}{16 \pi \chi_{3}}\right]>\bar{w}_{0 L}$. Therefore, the quantity $\alpha=\tilde{\varepsilon} / 8 \pi \chi_{3}$ can be represented as order parameter of the field-medium system: when $\alpha<0$, the symmetry of the system in the energy space breaks and the ground (vacuum) state degenerates for the field component amplitudes in the nonlinear propagation mode.

Assuming that the permittivity varies only slightly over the spatial scale of the wavelength, $\lambda|\nabla \ln \varepsilon|<<1$, we can use a nonlinear equation for the classical quasimonochromatic electric field $\mathbf{E}$,

$$
\left(\nabla^{2}-\frac{\varepsilon_{1}}{c^{2}} \frac{\partial^{2}}{\partial t^{2}}\right) \mathrm{E}=\frac{4 \pi \chi_{3}}{c^{2}} \frac{\partial^{2}}{\partial t^{2}}|\mathbf{E}|^{2} \mathbf{E},
$$

where $\nabla=\mathbf{1}_{x} \partial / \partial x+\mathbf{1}_{y} \partial / \partial y+\mathbf{1}_{z} \partial / \partial z$ is the nabla operator, $\mathbf{1}_{x, y, z}$ the unit vectors, $\varepsilon_{1}=1+4 \pi \chi_{1}$ and $\chi_{1}$ and $\chi_{3}$ respectively the linear and cubic susceptibilities of the medium. Eq. (11) describes propagation of the nonlinear waves in both an infinite dielectric medium and a dielectric waveguide.

The permittivity of the medium is a function of the field energy density $\left(\varepsilon=\varepsilon_{1}+4 \pi \chi_{3} \mathbf{E} * \mathbf{E}\right)$. For quasi-monochromatic fields, expanding the operator $f_{2}(\omega+\Delta \omega)=\frac{\partial^{2}}{\partial t^{2}} \varepsilon$ in Eq. (11) in the spectral width $\Delta \omega<<\omega$ with properly accounting dispersion properties of the medium yields a system of equations for slowly varying amplitudes $\psi_{j}(t, \mathbf{r})=\tilde{\psi}_{j}(\mathbf{r}) e^{-i \Delta \omega t}$ of the electric vector components $\mathbf{E}_{j}=\psi_{j}(t, \mathbf{r}) e^{-i \omega t}(\mathrm{j}=1,2$ and 3$)$ :

$$
\begin{array}{r}
\left(\nabla^{2}+\frac{\omega^{2} \varepsilon_{1}}{c^{2}}\right) \psi_{j}+i \frac{1}{c^{2}} \frac{d\left(\omega^{2} \varepsilon_{1}\right)}{d \omega} \frac{\partial \psi_{j}}{\partial t}+\omega^{2} \frac{4 \pi \chi_{3}}{c^{2}}|\psi|^{2} \psi_{j}+ \\
+i \omega \frac{8 \pi \chi_{3}}{c^{2}} \frac{\partial|\psi|^{2} \psi_{j}}{\partial t}-\frac{4 \pi \chi_{3}}{c^{2}} \frac{\partial^{2}|\psi|^{2} \psi_{j}}{\partial t^{2}}=0
\end{array}
$$

where $|\psi|^{2}=\psi_{j}^{*} \psi_{j}$. Assuming that $\left|\partial \psi_{j} / \partial t\right| \sim\left|\chi_{3}\right|$, we get from Eq. (12) a system of three nonlinear first-order equations for the field propagating in the medium,

$$
i \frac{\partial \psi_{j}}{\partial t}=-a_{0} \nabla^{2} \psi_{j}-a_{1} \psi_{j}-a_{2}|\psi|^{2} \psi_{j},
$$

with the notations $a_{0}=c^{2}\left[d\left(\omega^{2} \varepsilon_{1}\right) / d \omega\right]^{-1}, \quad a_{1}=\omega^{2} \varepsilon_{1}\left[d\left(\omega^{2} \varepsilon_{1}\right) / d \omega\right]^{-1} \quad$ and 


$$
a_{2}=4 \pi \omega^{2} \chi_{3}\left[d\left(\omega^{2} \varepsilon_{1}\right) / d \omega\right]^{-1} \text {. }
$$

The Lagrangian of the system of Eqs. (13) has the form

$$
L=i \psi_{j}\left(\partial \psi_{j}^{*} / \partial t\right)-i \psi_{j}^{*}\left(\partial \psi_{j} / \partial t\right)+a_{0}\left(\nabla \psi_{j}^{*}\right) \nabla \psi_{j}-a_{1} \psi_{j}^{*} \psi_{j}-\frac{a_{2}}{2}\left(\psi_{j}^{*} \psi_{j}\right)^{2}-\text { const. }
$$

If the field is described by the potential $U(\psi)=a_{1} \psi_{j}^{*} \psi_{j}+\frac{a_{2}}{2}\left(\psi_{j}^{*} \psi_{j}\right)^{2}+$ const, with $a_{1}>0$ and $a_{2}>0$, the system has a stable symmetric state at $\psi=\psi_{V 0}$ (see Fig. 2, curve 1). However, if $a_{1}<0$ and $a_{2}>0$, one has $d\left(\omega^{2} \varepsilon_{1}\right) / d \omega<0$ and $\chi_{3}<0$ and the state at $\psi=\psi_{V 0}$ is symmetric though unstable. At $\psi_{V j} \neq 0$, there arises a stable vacuum state with the broken symmetry: $\left|\psi_{V}\right|^{2}=\varepsilon_{1} / 4 \pi\left|\chi_{3}\right|$, where $\left|\psi_{V}\right|^{2}$ is the polariton condensate density (Fig. 2, curve 2). If $a_{1}>0$ and $a_{2}<0 \quad\left(\chi_{3}<0\right)$, the potential corresponds to the attraction of polaritons (Fig. 2, curve 3).

Consider now the propagation of non-monochromatic field in a boundless medium. Eq. (14) for this case yields a nonlinear dispersion relation for the quasi-plane waves $\psi_{j}=\psi_{0 j} \exp [-i \Delta \omega t+i \mathbf{k r}]$ :

$$
\left(\varepsilon_{1}+\Delta \omega \frac{d \varepsilon_{1}}{d \omega}+4 \pi \chi_{3} \sum \psi_{0 j}^{2}\right) \omega^{2}+2 \Delta \omega \varepsilon_{1} \omega=c^{2} k^{2} .
$$

From the dispersion relation (15), we obtain the two-branch frequency spectrum,

$$
\omega_{N L}=-\Delta \omega \varepsilon_{1} / \varepsilon_{N L} \pm \sqrt{\left(\Delta \omega \varepsilon_{1}\right)^{2} / \varepsilon_{N L}^{2}+c^{2} k^{2}},
$$

where $\varepsilon_{N L}=\varepsilon_{1}+\Delta \omega \frac{d \varepsilon_{1}}{d \omega}+4 \pi \chi_{3} \sum \psi_{0 j}^{2}$. The frequencies given by Eq. (16) acquire the values $\omega_{+}=0$ and $\omega_{-}=-2 \Delta \omega \varepsilon_{1} / \varepsilon_{N L}$ if $k \rightarrow 0$. The quasi-particle energy is given by $\tilde{E}=\hbar\left(\omega_{N L}+\Delta \omega \varepsilon_{1} / \varepsilon_{N L}\right)=\left[\left(\hbar \Delta \omega \varepsilon_{1}\right)^{2} / \varepsilon_{N L}^{2}+c^{2} \hbar^{2} k^{2}\right]^{1 / 2}$. Thus, a massless Goldstone boson $\left(m_{G B}=0\right) \quad$ and a quasi-particle with the mass $m_{P}=c^{-2} \hbar\left[\left(\omega_{N L}+\Delta \omega \varepsilon_{1} / \varepsilon_{N L}\right)^{2}-c^{2} k^{2}\right]^{1 / 2}=c^{-2} \hbar \Delta \omega \varepsilon_{1} / \varepsilon_{N L}$ are generated in the system. Notice that the generation of massless boson and a massive quasi-particle in the nonlinear mode of the field-medium interaction is a consequence of spontaneous symmetry breaking in the energy domain $[3,10]$ under the condition $\alpha<0$. In the long run, the symmetry of field-medium system spontaneously breaks if the medium reveals nonlinear effects and the appropriate number $\mathrm{N}$ of photons is available in the given region of that medium (since $|\psi|^{2} \sim N \tilde{E}_{1}$ ).

In the unclosed Fabry-Perot resonator, the type of translational symmetry along the longitudinal axis $\mathrm{z}$ differs from that peculiar for the transverse plane because of a 


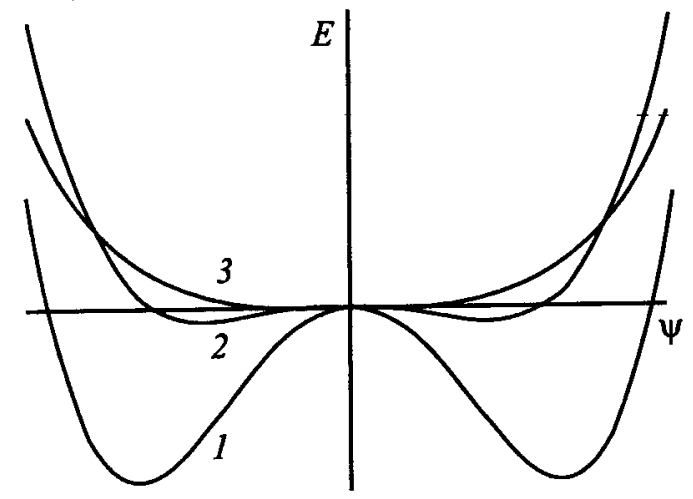

Fig. 2. Profiles of the internal energy $\tilde{E}_{I}$ calculated for $m_{\ell}=1, \ell=1, \alpha=0.1$, and the cases of $R=0.4$ (1), $R=0.5$ (2) and $R=1$ (3).

presence of interfaces in the $\mathrm{z}$ direction. If the resonator is filled with a dispersive insulator, the squared wave number may be represented as $k^{2}=k_{\perp}^{2}+\beta^{2}$, whith $\beta=\ell \pi / L$ being the propagation constant along the $\mathrm{z}$ axis $(\ell=1,2, \ldots)$ and $\mathrm{L}$ the resonator length. Then the frequency (16) of the $\ell$ th mode becomes

$$
\omega_{\ell}=-\Delta \omega \varepsilon_{1} / \varepsilon_{N L} \pm \sqrt{\left(\Delta \omega \varepsilon_{1}\right)^{2} / \varepsilon_{N L}^{2}+c^{2} k_{\perp}^{2}+c^{2} \ell^{2} \pi^{2} / L^{2}} .
$$

When $k_{\perp} \rightarrow 0$, one has $\omega_{\ell} \neq 0$ and so can determine easily the masses of quasiparticles referred to as polaritons: $m_{\ell}=c^{-2} \hbar\left[\left(\omega_{\ell}+\Delta \omega \varepsilon_{1} / \varepsilon_{N L}\right)^{2}-c^{2} k_{\perp}^{2}\right]^{1 / 2}$. The polaritons in the dielectric resonator acquire a mass in both the linear $\left(\chi_{3}=0\right)$ and nonlinear $\left(\chi_{3}>0\right.$ or $\left.\chi_{3}<0\right)$ propagation modes. The polariton mass depends upon the amount of response from the medium. The polariton takes a mass due to constraints imposed on its longitudinal motion (a one-dimensional potential well). In terms of classical electrodynamics, this means that a standing wave arises in the resonator (i.e., between its mirrors). The standing wave does not transfer the field energy; one can therefore determine the polariton mass in the laboratory coordinate system.

Let us find the mass spectrum of quasi-particles in the dielectric waveguide. Since the spectrum of the propagation constants $\beta_{\ell}$ is discrete, the electromagnetic field travelling in the waveguide may be considered as a flow of massive quasi-particles. The waveguide imposes constraints on the radial degree of freedom, without disturbing the longitudinal (along the $\mathrm{z}$ axis) and azimuthal degrees of freedom. Thus, the massive quasi-particles appearing in the waveguide are due to radial constraints (interfaces).

The system of Eqs. (13) for the quasi-monochromatic field turns into

$$
\nabla^{2} \psi_{j}+\left(\Delta \omega \frac{\partial\left(\omega^{2} \varepsilon_{1}\right)}{c^{2} \partial \omega}+\frac{\omega^{2} \varepsilon_{1}}{c^{2}}\right) \psi_{j}-\frac{4 \pi \chi_{3} \omega^{2}}{c^{2}}|\psi|^{2} \psi_{j}=0
$$

The system (18) splits in the linear mode $\left(\chi_{3}=0\right)$. In the cylindrical coordinate system, the equation for the field components may be written as 


$$
\left(\frac{\partial^{2}}{\partial r^{2}}+\frac{1}{r} \frac{\partial}{\partial r}+\frac{1}{r^{2}} \frac{\partial^{2}}{\partial \varphi^{2}}+\frac{\partial^{2}}{\partial z^{2}}\right) \psi_{j}+\left(\Delta \omega \frac{\partial\left(\omega^{2} \varepsilon_{1}\right)}{c^{2} \partial \omega}+\frac{\omega^{2} \varepsilon_{1}}{c^{2}}\right) \psi_{j}=0 .
$$

This linear equation takes a standard Bessel form if the field components $\psi_{j}=\psi_{j}(r) \exp (-i \kappa \ell \varphi+i \beta z)$ are sought:

$$
\left(\frac{\partial^{2}}{\partial r^{2}}+\frac{1}{r} \frac{\partial}{\partial r}\right) \psi_{j}+\left(u^{2}-\frac{\ell^{2}}{r^{2}}\right) \psi_{j}=0
$$

with $u^{2}=\Delta \omega \frac{\partial\left(\omega^{2} \varepsilon_{1}\right)}{c^{2} \partial \omega}+\frac{\omega^{2} \varepsilon_{1}}{c^{2}}-\beta^{2}$. Its solutions for the guided modes might be represented as the Bessel functions of the first kind, $\psi_{j}(r)=A J_{\ell}(u r)$. If the field is confined in the circular waveguide with the radius $R_{0}$, the dispersion relation becomes $J_{\ell}\left(u R_{0}\right)=0$. Denoting its roots by $M_{\ell n}(\mathrm{n}=1,2, \ldots)$ and squaring the dispersion equation $u R_{0}=M_{\ell n}$, we derive the dispersion law:

$$
\omega^{2} \varepsilon_{1}+\Delta \omega \frac{\partial\left(\omega^{2} \varepsilon_{1}\right)}{\partial \omega}=c^{2} M_{\ell n}^{2} / R_{0}^{2}+c^{2} \beta_{\ell n}^{2}
$$

Taking a physically meaningful solution of Eq. (21) $(\omega>0)$,

$$
\omega_{\ell n+}=-\frac{\Delta \omega \varepsilon_{1}}{\varepsilon_{1}+\Delta \omega \partial \varepsilon_{1} / \partial \omega}+\sqrt{\left(\frac{\Delta \omega \varepsilon_{1}}{\varepsilon_{1}+\Delta \omega \partial \varepsilon_{1} / \partial \omega}\right)^{2}+c^{2} \beta_{\ell n}^{2}+c^{2} M_{\ell n}^{2} / R_{0}^{2}},
$$

one can find from Eq. (22) the mass spectrum of polaritons in the waveguide for the linear mode:

$$
m_{\ell n+}=c^{-2} \hbar\left[\left(\omega_{\ell n+}+\frac{\Delta \omega \varepsilon_{1}}{\varepsilon_{1}+\Delta \omega \partial \varepsilon_{1} / \partial \omega}\right)^{2}-c^{2} \beta_{\ell n}^{2}\right]^{1 / 2} .
$$

With the polaritons inside, the waveguide becomes heavier. The mass of the waveguide with the field is $m_{W}=m_{0 W}+\sum m_{\ell n}$, where $m_{0 W}$ is the waveguide mass without taking the field into account. Here the summation is performed over the number of field modes and the number of quasi-particles per mode, the latter being defined by the squared mode amplitude. For example, if the lowest mode $H E_{11}\left(M_{11}=4\right)$ of radiation with the wavelength $\lambda=0.6328 \mu \mathrm{m}$ in air propagates through the quartz fibre (the core radius $R_{0}=3 \mu \mathrm{m}$ ) with the propagation constant $\beta_{11} \approx 1.45 \cdot 10^{5} \mathrm{~cm}^{-1}$, the mass of the quasi-particle excited in the fibre is $m_{11} \sim 5 \cdot 10^{-34} \mathrm{~g}$.

Considering the polaritons as excitations of the vacuum state in the nonlinear mode $\left(\left|\psi_{V}\right|^{2}=\varepsilon_{1} / 4 \pi\left|\chi_{3}\right|\right)$, we linearize Eq. (18) (in this case $\psi_{j}=\sqrt{\varepsilon_{1} / 12 \pi\left|\chi_{3}\right|}+\eta_{j}$ and 
$|\psi|^{2}=\varepsilon_{1} / 4 \pi\left|\chi_{3}\right|+|\eta|^{2}$ ) and obtain the following equation:

$$
\nabla^{2} \eta_{j}+\Delta \omega \frac{\partial\left(\omega^{2} \varepsilon_{1}\right)}{c^{2} \partial \omega} \eta_{j}+\Delta \omega \frac{\partial\left(\omega^{2} \varepsilon_{1}\right)}{c^{2} \partial \omega} \sqrt{\frac{\varepsilon_{1}}{12 \pi\left|\chi_{3}\right|}}=0 .
$$

Renormalizing the polariton amplitude $\left(\bar{\eta}_{j}=\eta_{j}+\sqrt{\varepsilon_{1} / 12 \pi\left|\chi_{3}\right|}\right)$, we come to the linearized equation

$$
\left(\frac{\partial^{2}}{\partial r^{2}}+\frac{1}{r} \frac{\partial}{\partial r}+\frac{1}{r^{2}} \frac{\partial^{2}}{\partial \varphi^{2}}+\frac{\partial^{2}}{\partial z^{2}}\right) \bar{\eta}_{j}+b^{2} \bar{\eta}_{j}=0
$$

with $b^{2}=\Delta \omega \frac{\partial\left(\omega^{2} \varepsilon_{1}\right)}{c^{2} \partial \omega}$. Eq. (25) has the solution $\bar{\eta}_{j}=A J_{\ell}(u r) \exp (-i \kappa \ell \varphi+i \beta z)$, where $\bar{u}^{2}=b^{2}-\beta^{2}$ and $\kappa= \pm 1$. Assuming that the field is confined within the waveguide $\left(\bar{\eta}_{j}\left(\bar{u} R_{0}\right)=0\right)$ and using the dispersion relation $J_{\ell}\left(\bar{u} R_{0}\right)=0$, we obtain the dispersion law in the form

$$
\Delta \omega \frac{\partial\left(\omega^{2} \varepsilon_{1}\right)}{\partial \omega}=c^{2} M_{\ell n}^{2} / R_{0}^{2}+c^{2} \beta_{\ell n}^{2}
$$

and, hence, the frequency spectrum,

$$
\omega_{\ell n+}=-\frac{\Delta \omega \varepsilon_{1}}{\partial \varepsilon_{1} / \partial \omega}+\sqrt{\left(\frac{\Delta \omega \varepsilon_{1}}{\partial \varepsilon_{1} / \partial \omega}\right)^{2}+c^{2} \beta_{\ell n}^{2}+c^{2} M_{\ell n}^{2} / R_{0}^{2}} .
$$

Now, having Eq. (27) substituted into Eq. (23), we derive the mass spectrum of polaritons in the waveguide for the nonlinear interaction mode. Comparing the frequency spectra given by Eqs. (22) and (27), one can see that the linear polaritons are lighter in weight than the nonlinear ones, provided that the order of dispersion $\partial \varepsilon_{1} / \partial \omega$ in the medium is the same.

It is well known that the mass of particle depends on its binding energy [11]. In frame of the models considered above, the polariton mass is specified by interaction between the electromagnetic field and the dielectric medium. In this case the massive quasi-particle cannot move with the vacuum speed of light, either in boundless medium or in waveguide, as supported by our results. For instance, the velocity of polariton in the nonlinear waveguide

$$
v_{\ell n}=\frac{d \omega_{+}}{d \beta_{\ell n}}=\frac{c^{2} \beta_{\ell n}}{\sqrt{\left(\frac{\Delta \omega \varepsilon_{1}}{\partial \varepsilon_{1} / \partial \omega}\right)^{2}+c^{2} \beta_{\ell n}^{2}+c^{2} M_{\ell n}^{2} / R_{0}^{2}}}<c
$$

is evidently lower than the vacuum light speed. 


\section{Three-dimensional quasi-particles in dielectric waveguide}

Let us consider the propagation of electromagnetic field in isotropic dielectric waveguide possessing the shape of circular cylinder [9]. Taking into account the cubic response of the waveguide medium (e.g., the quartz), one can see that the nonlinear equation for the electric field strength $\mathbf{E}$ is represented by Eq. (11). Assuming that $\mathbf{E}$ is a harmonic function of time (i.e., ignoring the harmonics generation effects), we can present the field in the form of waveguide modes with the real amplitudes $e(\mathbf{r})$ :

$$
\mathbf{E}=\mathrm{e}(\mathbf{r}) \exp (i \kappa \ell \varphi) \exp [i(\omega t-\beta z)], \quad(\kappa= \pm 1, \ell=0,1,2, \ldots) .
$$

Then substituting Eq. (29) into Eq. (11) results in the equation system written in the cylindrical coordinates:

$$
\left(\frac{d^{2}}{d R^{2}}+\frac{1}{R} \frac{d}{d R}-\frac{\ell^{2}}{R^{2}}\right) e_{j}+m^{2} e_{j}+\alpha e^{2} e_{j}=0,(\mathrm{j}=\mathrm{x}, \mathrm{y}, \mathrm{z}),
$$

where $m^{2}=r_{0}^{2}\left(\omega^{2} \varepsilon_{1} / c^{2}-\beta^{2}\right), \alpha=4 \pi \chi_{3} r_{0}^{2} \omega^{2} / c^{2}, e^{2}=\sum_{j=x, y, z} e_{j}^{2}, R=r / r_{0} \in[0,1]$ and $r_{0}$ is the waveguide radius. The mode propagation constants $\beta$ may be determined from dispersion equation [15]. Note that the field equation (30) has the form of nonlinear steady-state Klein-Gordon equation. This implies that the electromagnetic field propagating in the waveguide may be considered, in view of discreteness of the spectrum of propagation constants $\beta_{\ell}$, as a flux of quasi-particles possessing the effective "dimensionless" masses $m_{\ell}=r_{0} \sqrt{\omega^{2} \varepsilon_{1} / c^{2}-\beta_{\ell}^{2}}$.

On substituting $e_{j}=\psi_{j}(r) / \sqrt{R}$, the system of Eqs. (30) may be rewritten in the form of equations describing a motion of quasi-particle under the action of central force:

$$
m_{\ell} \psi_{x}^{\prime \prime}=-\frac{\partial U_{\ell}}{\partial \psi_{x}}, m_{\ell} \psi_{y}^{\prime \prime}=-\frac{\partial U_{\ell}}{\partial \psi_{y}}, m_{\ell} \psi_{z}^{\prime \prime}=-\frac{\partial U_{\ell}}{\partial \psi_{z}},
$$

where the prime indicates differentiation with respect to $\mathrm{R}$,

$$
U_{\ell}=\frac{m_{\ell}}{2}\left(m_{\ell}^{2}-\frac{\ell^{2}-1 / 4}{R^{2}}\right) \psi^{2}+\frac{\alpha m_{\ell}}{4 R}\left(\psi^{2}\right)^{2}
$$

is the potential and $\psi^{2}=\psi_{x}^{2}+\psi_{y}^{2}+\psi_{z}^{2}$. The Hamiltonian of this system is as follows:

$$
H=\frac{1}{2} \psi^{\prime 2}+\frac{1}{2}\left(m_{\ell}^{2}-\frac{\ell^{2}-1 / 4}{R^{2}}\right) \psi^{2}+\frac{\alpha}{4 R}\left(\psi^{2}\right)^{2} .
$$

In the configuration space, the first term describes the "kinetic" energy (or, in the real space, the potential energy) that depends on the rate of spatial variation of the field strength, while the second and the third terms describe the internal energy of the system. 
Let us determine the states of the system characterized by the condition $\mathrm{H}=$ const. The ground state (with the zero potential energy (32), where by $\psi^{\prime 2} / 2=0$ ) corresponds to the internal energy

$$
\tilde{E}_{I}=\frac{\alpha}{4 R}\left(\psi^{2}\right)^{2}+\frac{1}{2}\left(m_{\ell}^{2}-\frac{\ell^{2}-1 / 4}{R^{2}}\right) \psi^{2} .
$$

The potential energy is positive in the excited state, so that the vacuum states can take place only when the internal energy is zero $\left(\tilde{E}_{I}=0\right)$. This yields the equation for the field components in the vacuum states:

$$
\psi^{4}-\frac{2 R}{\alpha}\left(m_{\ell}^{2}-\frac{\ell^{2}-1 / 4}{R^{2}}\right) \psi^{2}=0,
$$

which has its solutions lying on the spheres with the square radius

$$
\psi_{\ell}^{2}=\frac{2 R}{\alpha}\left(m_{\ell}^{2}-\frac{\ell^{2}-1 / 4}{R^{2}}\right)>0 .
$$

On the waveguide axis $(\mathrm{R}=0)$, all the field components are zero at $\tilde{E}_{I}=0$ : $\psi_{x}=\psi_{y}=\psi_{z}=0$.

In the system manifesting more than one vacuum state, the transitions among those states can take place without any energy dissipation, whereby the field acquires the form of a solitary wave (soliton) [5]. At the same time, it is known that the soliton solutions correspond to the system trajectory of the phase plane representing separatrix that passes through the points $\psi_{S}^{\prime}=0$ and $d U_{\ell}\left(\psi_{S}\right) / d \psi=0$ [17]. The equation

$$
\frac{d U_{\ell}}{d \psi}=\psi_{S}^{3}+\frac{R}{\alpha}\left(m_{\ell}^{2}-\frac{\ell^{2}-1 / 4}{R^{2}}\right) \psi_{S}=0
$$

has the solutions

$$
\psi_{S}=0, \psi_{S \ell}=\sqrt{\frac{R}{\alpha}\left(\frac{\ell^{2}-1 / 4}{R^{2}}-m_{\ell}^{2}\right)} .
$$

Substituting $\psi_{S \ell}$ into Eq. (33), we determine the system energies corresponding to the soliton solutions:

$$
H_{S}=-\frac{m_{\ell} R}{4 \alpha}\left(m_{\ell}^{2}-\frac{\ell^{2}-1 / 4}{R^{2}}\right)^{2} .
$$

Fig. 2 shows the shape of the potential $\tilde{E}_{I}(\psi, R)$ for different values of the parameter R. As seen from Fig. 2, the form of the potential $\tilde{E}_{I}$ depends on the proximity 


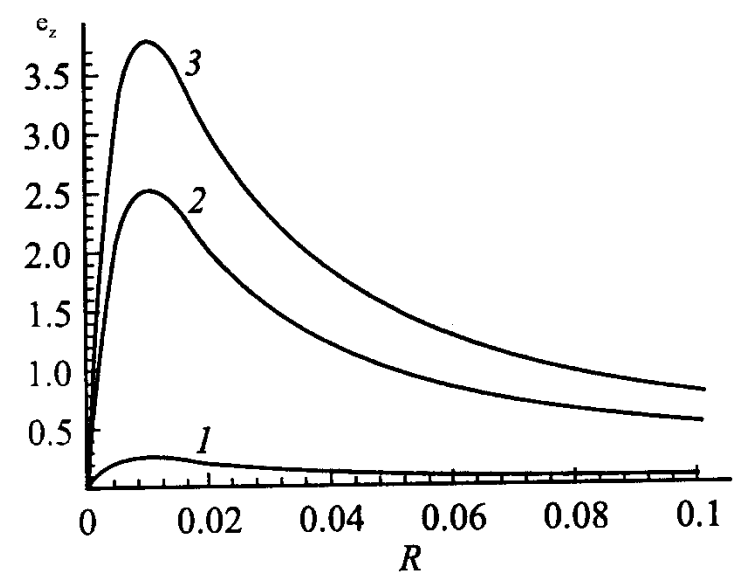

Fig. 3. Profiles of the electric field components calculated for $m_{\ell}=1, \ell=1$ and $\alpha=0.1$ : (1) $e_{z}$ for $e_{z}(0)=0.01,(2) \mathrm{e}_{\mathrm{x}}$ for $e_{x}(0)=0.1$ and (3) $e_{y}$ for $e_{y}(0)=0.15$.

of boundaries (i.e., on the parameter $\mathrm{R}$ representing the normalized waveguide radius). The solutions for the field components $\left(e_{x}, e_{y}\right.$ and $\left.e_{z}\right)$ of the solitary wave, which are obtained upon solving Eqs. (30) with numerical methods, are presented in Fig. 3. The potential exhibits a characteristic shape favouring spontaneous breaking of the system symmetry and, hence, making particle-like solutions possible in the region far from the boundaries $(R \neq 0)$.

\section{Conclusion}

Thus, the mass of a quasi-particle arises because it moves in the medium or the waveguide with the velocity less than the speed of light in vacuum. This is a result of interaction of the electromagnetic field and the medium. The "electromagnetic fielddielectric waveguide" system reveals a complicated topological structure characterized by a presence of degenerate vacuum states with zero energy. These regions are formed depending on the system energy. The presence of degenerate vacuum states evidences that the electromagnetic field in the dielectric waveguide can exist in the form of threedimensional massive quasi-particles, thus manifesting the properties of particles. Besides, the mass of quasi-particles occurs due to spontaneous breaking of the system symmetry, which is caused by a presence of boundaries or nonlinear interaction of polaritons in the boundless dielectric medium.

\section{References}

1. Klyshko D.N. Photons and Nonlinear Optics. Moscow : Nauka. (1980).

2. Davydov A.S. Theory of Solids. Moscow: Nauka. (1976).

3. Ryder L.H. Quantum Field Theory, 2nd ed. Cambridge: Cambridge Univ. Press. (1996). 
4. Polyakov AM, 1974. Spectrum of particles on quantum field theory. Pisma ZhETF. 20: 430-433 [JETP Lett. 20, 194 (1974)].

5. Rebbi K, 1980. Solitons. Usp.Fiz.Nauk 130: 329-356 [1980. Sov.Phys. Usp. 23: 1].

6. Belova TI and Kudryavtsev AE, 1997. Solitons and their interaction on the classical field theory. Usp.Fiz.Nauk 167: 377-406 [1997. Sov.Phys. Usp. 40: 369].

7. Rivlin LA, 1997. Photons in waveguide (several imagine experiments). Usp.Fiz.Nauk 167: 309-322 [1997. Sov.Phys. Usp. 40: 291].

8. Kivshar Y.S. and Agraval G.P., Optical Solitons. From Fibers to Photonic Crystals. New York: Academic Press (2003).

9. Dzedolik IV, 2003. The topology of "vacuum" in a dielectric waveguide. Pisma Zh.Tekh.Fiz. 29: 42-47 [2003. Tech. Phys. Lett. 29: 284].

10. Dzedolik IV, 2006. Spontaneous symmetry breaking in an electromagnetic field insulator system. Zh.Tekh.Fiz. 76: 116-120 [2006. Tech. Phys. Lett. 51: 932].

11. Okun LB, 1989. Conception of mass. Usp.Fiz.Nauk 158: 511-530 [1989. Sov.Phys. Usp. 32: 629].

12. Gabovich AM and Gabovich NA, 2007. How to explain the non-zero mass of electromagnetic radiation consisting of zero-mass photon. European J.Phys. 28: 649655.

13. De-Broglie L. Electromagnetic waves in hollow waveguides and resonators. Moscow: GIIL (1948).

14. Feynman R.P., Leighton R.B. and Sands M. The Feynman Lectures on Physics, Vol. 2. Reading, Massachusetts, Palo Alto, London: Addison-Wesley Publishing Company, Inc. (1964).

15. Snayder A.W. and Love J.D. Optical Waveguide Theory. New York: Chapman and Hall, London (1983).

16. Landau L.D. and Lifshitz E.M. Course of Theoretical Physics, Vol. 8: Electrodynamics of Continuous Media. Moscow: Nauka (1982); New York: Pergamon (1984).

17. Zaslavskii G.M. and Sagdeev R.Z. Nonlinear Physics: from the Pendulum to Turbulence and Chaos. Moscow: Nauka (1987). 